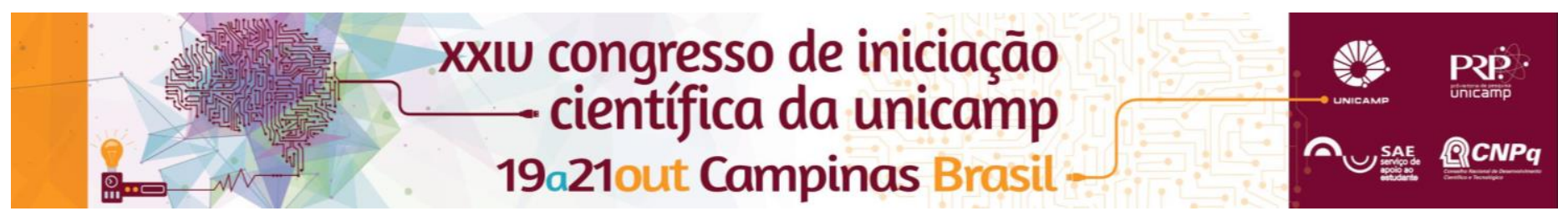

\title{
“Efeito da fumaça de diferentes origens fumígeras nas propriedades cromáticas e físicas de resinas compostas"
}

\author{
Lorena B. Costa*, Jéssica D. Theobaldo, Waldemir F. Vieira-Júnior, Débora A.N.L. Lima, Flávio H.B. Aguiar.
}

\section{Resumo}

Este estudo tem por objetivo avaliar se a exposição de diferentes resinas compostas à fumaça de cigarro de diferentes origens fumígeras é capaz de alterar propriedades físicas, clinicamente importantes, como cor e rugosidade. Foram utilizados neste estudo doi tipos de resina composta $(n=10)$ : microhíbrida (Z250, 3M ESPE, G1 - G5) e nanoparticulada (Z350XT, 3M ESPE, G6 - G10). As amostras foram expostas à fumaça de 1 maço de cigarro por dia/5dias: Marlboro Red (MR: G2 e G7), Marlboro Silver Light (MBSL: G3 e G8), Marlboro Ble Ice (MBl: G4 e G9) e Marlboro Filter Plus (MFP: $\mathrm{G} 5$ e $\mathrm{G} 10)$. As leituras de $\operatorname{cor}(\Delta \mathrm{E}, \Delta \mathrm{L}, \Delta \mathrm{a}$ e $\Delta \mathrm{b})$ e rugosidade (Ra) foram realizadas nos tempos: inicial (baseline) e final (após exposição à fumaça de cigarro). Os dados obtidos foram submetidos ao teste estatístico ANOVA 2 fatores e Tukey. Para todas as análises os grupos controles não diferiram entre si. Todos os tipos de fumaça de cigarro promoveram alteração de cor em ambas as resinas, alterando todos os parametros de cor analisados. A resina Z350 foi mais susceptível ao manchamento quando comparada à Z250. A rugosidade foi alterada somente na resina Z350 quando exposta ao MBI. A resina Z350 é mais susceptível ao manchamento por cigarro, independentemente do tipo de fumaça à que foi exposta. A fumaça de cigarro Marlboro Blue lce, por conter essências, tem maior potencial efeito de manchamento para resina Z250.

\section{Palavras-chave:}

Cor, rugosidade, fumaça de cigarro.

\section{Introdução}

As resinas compostas são os materiais de primeira escolha para a realização de restaurações dentais diretas, principalmente em regiões anteriores, devido suas características estéticas ${ }^{1}$. A fumaça do cigarro é uma complexa matriz constituída por uma fase gasosa e outra particulada, sendo esta última, constituída principalmente por alcatrão, substância de coloração amarelo - amarronzada, que tem sido associada ao manchamento da estrutura dental e resinas compostas ${ }^{2}$.

O objetivo deste trabalho foi avaliar o efeito da exposição de diferentes resinas compostas à fumaça de cigarro de diferentes origens fumígeras nas propriedades de cor e rugosidade.

\section{Resultados e Discussão}

A análise de variação de cor $(\Delta \mathrm{E})$ demonstrou efeito para os fatores: resina $(p<0,0001)$, cigarro $(p<0,0001)$ e para interação entre os fatores. Os grupos sem exposição à fumaça de cigarro não diferiram em relação à resina. Quando comparamos as resinas entre si, para todos os tipos de cigarro, a variação de cor na resina Z350 foi significativamente maior quando comparada a Z250.

Tabela 1: Média (desvio padrão) da variação de cor $(\Delta \mathrm{E})$ de resinas compostas expostas à fumaça de diferentes cigarros.

\begin{tabular}{ccc}
\hline & \multicolumn{2}{c}{ Resina } \\
\cline { 2 - 3 } Sem exposição & $0.62(0.24) \mathrm{Ac}$ & $0.94(0.21) \mathrm{Ac}$ \\
MR & $2.06(0.72) \mathrm{Bb}$ & $4.72(0.83) \mathrm{Aa}$ \\
MSL & $2.53(0.31) \mathrm{Bab}$ & $4.8(0.64) \mathrm{Aa}$ \\
MBI & $3.25(0.42) \mathrm{Ba}$ & $4.6(0.91) \mathrm{Aa}$ \\
MFP & $2.40(0.38) \mathrm{Bb}$ & $3.21(0.56) \mathrm{Ab}$ \\
\hline
\end{tabular}

Letras distintas (maiúscula em horizontal e minúscula na vertical) são estatisticamente diferentes $(\mathrm{p}<0,05)$. MR: Marlboro Red; MSL: Marlboro Silver Light; MBI: Marlboro Blue Ice; MFP: Marlboro Filter Plus.

Conforme resultados apresentados na Tabela 1 , para resina microhíbrida Z250, todos os grupos expostos à fumaça de cigarro diferiram estatisticamente do grupo controle, apresentando uma maior alteração de cor. Dentre os cigarros $\mathrm{MBI}$ apresentou os maiores valores sendo estatisticamente diferente de MR e MFP. MSL apresentou valores intermediários não diferindo dos outros tipos de cigarro.

Para resina nanoparticulada Z350, todos os grupos expostos à fumaça de cigarro diferiram estatisticamente do grupo controle, apresentando uma maior alteração de cor. Dentre os cigarros MFP foi o que apresentou menor alteração de cor diferindo estatisticamente de MR, MSL e $\mathrm{MBI}$ que por sua vez não diferiram entre si.

Para $\Delta \mathrm{L}, \Delta \mathrm{a}$ e $\Delta \mathrm{b}$ a resina $\mathrm{Z350}$ apresentou maiores variações, diferindo estatisticamente da resina Z250, independentemente do tipo de cigarro.

Para $\Delta \mathrm{Ra}, \quad \mathrm{Z} 250$ e Z350 não diferiram estatisticamente, mesmo após a exposição fumaça de cigarro. Para Z350, MBI apresentou maior variação de rugosidade, apresentando diferença estatística em relação ao controle, MFP e MR. MSL apresentou valores intermediários não diferindo dos demais grupos.

\section{Conclusões}

A resina $Z 350$ é mais susceptível ao manchamento por cigarro, independentemente do tipo de fumaça à que foi exposta. A fumaça de cigarro Marlboro Blue Ice tem maior potencial efeito de manchamento para resina Z250.

\section{Agradecimentos}

Este estudo foi financiado pelo CNPQ - PIBIC.

1, Heintze SD, Rousson V, Hickel R. Clinical effectiveness of direct anterior restorations-A meta-analysis. Dental Materials; in press.

2. Watts A, Addy M (2001) Tooth discolouration and staining: a review the literature. British Dental Journal; 190: 309-16.

3. Ferracane JL. Resin composite--state of the art. Dental Materials, 2001; 27(1): 29-38. 